\title{
Adult deterioration detection system (q-adds) based rapid response system (rrs) reduces severity of illness and length of stay of icu admissions from the ward in a regional hospital
}

KS Joshi, VK Campbell*, RJ Gooch, CM Anstey, MD Landy

From ESICM LIVES 2015

Berlin, Germany. 3-7 October 2015

\section{Introduction}

Hospital systems for the recognition (afferent limb) and management (efferent limb) of deteriorating patients, or Rapid Response Systems (RRS), are being mandated world-wide. This is despite conflicting evidence regarding their efficacy, with studies criticised for afferent limb failure, and questionable end points1. Additionally there are concerns regarding treatment limitation decisions and ward staff deskilling. Still, as elsewhere, we were admitting patients into the Intensive Care Unit (ICU) who we felt we should have been involved with earlier.

\section{Methods}

Our retrospective, single-center, observational study compared equivalent 18 -month periods before-and-after the revised RRS. Revisions included the implementation of the Q-ADDS observation form [2], sustained staff education and feedback, mandatory call activation, and primary treating team leadership or co-leadership. Primary end points included RRS activation volume, improved illness severity of unplanned ICU admissions from the ward, ICU LOS and ICU mortality. Secondary end points included unplanned ICU admissions, cardio-respiratory arrests and hospital mortality.

\section{Results}

With the new RRS, APACHE II (21 to $17, \mathrm{p}<0.001$ ), APACHE III (68 to 64, $\mathrm{p}=0.011$ ) and SAPS (38 to $35, \mathrm{p}=$ $0.044)$ scores at ICU admission from the ward were reduced. Less patients were in the severest quartile of

Sunshine Coast Hospital and Health Service, Intensive Care Unit, Nambour, Australia

(c) 2015 Joshi et al.; This is an Open Access article distributed under the terms of the Creative Commons Attribution License (http:// creativecommons.org/licenses/by/4.0), which permits unrestricted use, distribution, and reproduction in any medium, provided the original work is properly cited.
APACHE II (17.5 to 6.5, $\mathrm{p}<0.001)$, APACHE III (15.5 to $7.5, \mathrm{p}=0.012)$ and SAPS (24 vs $14, \mathrm{p}=0.006)$. Accordingly, ICU LOS ( 4 Vs. 3 days, $\mathrm{p}=0.02$ ) was reduced and prolonged stay ( $>7$ days) also trended down (27\% to $19 \%$, $\mathrm{p}=0.055)$. Organ support initiation $(\mathrm{p}=0.3)$ and ICU mortality $(25 / 181(13.7 \%)$ vs. $33 / 239(13.8 \%), p=0.93)$ were unchanged, although the number were small. The frequency of RRS activation (11 Vs. 48/1000 admissions, $\mathrm{p}<0.001$ ) was increased, with only a $0.7 \%$ failure to initiate a response where indicated. Hospital mortality, albeit multifactorial, trended lower (1/57 Vs. 1/64 admissions, $\mathrm{p}=0.06)$. Unplanned ICU admissions and arrests remain unchanged.

\section{Conclusions}

This revised RRS has improved illness severity at ICU admission, reduced ICU length of stay in our hospital. These specific outcomes may provide a reasonable measure, of RRS efficacy, even in smaller centres.

Published: 1 October 2015

\section{References}

1. Hillman k, Cjen J, Cretikos M, Bellomo R, Brown D, Doig G, Finfer S, Flabouris A: MERIT study investigators: Introduction of the medical emergency team (MET) system: a cluster-randomised controlled trial. Lancet 2005, 365:2091-2097.

2. Preece Megan H. W, Mark S, Horswill, Hill Andrew, Watson Marcus O: The Development of Adult Deterioration Detection System (ADDS) Chart. 2010, May. Report prepared for the Australian Commission on Safety and Quality in Health Care's program for Recognising and Responding to Clinical Deterioration

doi:10.1186/2197-425X-3-S1-A141

Cite this article as: Joshi et al:: Adult deterioration detection system (q-adds) based rapid response system (rrs) reduces severity of illness and length of stay of icu admissions from the ward in a regional hospital. Intensive Care Medicine Experimental 2015 3(Suppl 1):A141. 